\title{
Harris Extended Power Lomax Distribution: Properties, Inference and Applications
}

\author{
Adebisi Ade Ogunde ${ }^{1}$, Victoria Eshomomoh Laoye ${ }^{1}$, Ogbonnaya Nzie Ezichi ${ }^{1}$, Kayode Oguntuase Balogun ${ }^{2}$ \\ ${ }^{1}$ Biostatistics Unit, Department of Statistics University of Ibadan, Oyo State \\ ${ }^{2}$ Department of Statistics, Federal School of Statistics Ibadan \\ Corresponding author: Adebisi Ade Ogunde, Biostatistics Unit, Department of Statistics University of Ibadan, Oyo State
}

Received: May 10, 2021 Accepted: June 8, 2021 Online Published: June 21, 2021

doi:10.5539/ijsp.v10n4p77 URL: https://doi.org/10.5539/ijsp.v10n4p77

\begin{abstract}
In this work, we present a five-parameter life time distribution called Harris power Lomax (HPL) distribution which is obtained by convoluting the Harris-G distribution and the Power Lomax distribution. When compared to the existing distributions, the new distribution exhibits a very flexible probability functions; which may be increasing, decreasing, J, and reversed $\mathrm{J}$ shapes been observed for the probability density and hazard rate functions. The structural properties of the new distribution are studied in detail which includes: moments, incomplete moment, Renyl entropy, order statistics, Bonferroni curve, and Lorenz curve etc. The HPL distribution parameters are estimated by using the method of maximum likelihood. Monte Carlo simulation was carried out to investigate the performance of MLEs. Aircraft wind shield data and Glass fibre data applications demonstrate the applicability of the proposed model.
\end{abstract}

Keywords: Harris power Lomax distribution, Bonferroni curve, Lorenz curve, Renyl entropy, Monte Carlo simulation

\section{Introduction}

In the last decades, the Lomax distribution introduced by Lomax (1954), has been discovered to be very useful in several areas of applications most especially in applied sciences such as applications in flood, queue theory, internet traffic control, life testing, wind speed, sea waves and many others. The use of Lomax distribution is motivated by its heavy tail and simplicity in applications. It provides an alternative to the exponential-type distributions (Rayleigh, Weibull, exponential, Gompertz, pareto.), for further study see Bryson (1974). A random variable $X$ is said to be distributed according to Lomax distribution, if it distribution function is given by

$$
G^{l}(x ; a, k)=1-\left(1+\frac{x}{a}\right)^{-k}, x>0
$$

with $a, k>0$. Thus, $k$ is a shape parameter and $a$ is a scale parameter. The associated probability density function $(p d f)$ corresponding to (1) is given by

$$
g^{l}(x ; a, k)=\frac{k}{a}\left(1+\frac{x}{a}\right)^{-(k+1)}, x>0
$$

It should be noted that the $p d f$ of Lomax distribution is naturally a special case of some well-known distributions, and this includes Feller-Pareto, Pareto type II, Pareto type IV, Fisher distribution, and many others. However, the Lomax distribution is limited in applications as a result of some of its limitations, and this includes: Lack of flexibility, heavy tailed features, poor fits etc. when used to model real life data which exhibits non-monotonic, bathtub failure rate. Based on the afore-mentioned reasons various efforts have been made to generalize the Lomax distribution in other to induce flexibility into the distribution and also improve its fits for a better modeling capability. Among these are: the gamma Lomax distribution by Cordeiro and Ortega (2015), Marshall-Olkin Extended Lomax distribution by Ghitany et al. (2007), Al-Zahrani and Sagor (2014) developed and studied the Poisson Lomax distribution, the Exponential Lomax distribution was studied by El-Bassiouny et al. (2015), the Weibull Lomax distribution was studied by Tahir et al. (2015), Abdul-Moniem and Abdel-Hameed (2012) developed and studied the Exponentiated Lomax distribution, the transformed-transformer Lomax distribution was studied by Alzaghal and Hamed (2019), Kilany (2016) studied the weighted Lomax distribution, and the Power Lomax distribution was developed and studied by Rady et al. (2016).

For the purpose of this study, let us give a fair description of the Power Lomax $(P L)$ distribution. A random variable $X$ has a power Lomax distribution, if its cumulative distribution function $(c d f)$ and survival function is respectively 
given by

$$
F(x ; a, b, k)=G^{L}\left(x^{b} ; a, k\right)=1-\left(1+\frac{x^{b}}{a}\right)^{-k}, x>0
$$

And

$$
\bar{F}(x ; a, b, k)=G^{L}\left(x^{b} ; a, k\right)=\left(1+\frac{x^{b}}{a}\right)^{-k}, x>0
$$

With $a, b, k>0$. Thus, $b$ and $k$ are the shape parameters and $a$ is a scale parameter. The corresponding $p d f$ to (3) is given by

$$
f(x ; a, b, k)=g^{L}\left(x^{b} ; a, k\right)=\frac{b k}{a} x^{b-1}\left(1+\frac{x^{b}}{a}\right)^{-(k+1)}, x>0
$$

The additional parameter $b$ produces a tremendous effect on the flexibility of the $p d f$ of $P L$ distribution in such that the $p d f$ of $P L$ distribution is decreasing if $b \leq 1$ and increasing if $b>1$.

Using the Harris-G distribution proposed and developed by Aly and Benkherouf (2011), we develop the five-parameter distribution called Harris Power Lomax (HPL) distribution. Now, Considering a sequence of i.i.d random variables $Z_{1}, Z_{2}, \ldots, Z_{N}$ with cumulative distribution function $(c d f) G(x)$, probability density function ( $p d f$ ), hazard rate function $h(x)$ and Cumulative hazard rate function $R(x)$. Let $=\min \left(Z_{1}, Z_{2}, \ldots, Z_{N}\right)$, taking $N$ has a positive integer valued random variable with probability generating function $(p g f) \gamma(., c)$ for $\mathrm{c}>0$. The survival function $\bar{F}(x)$ of $X$ is given by

$$
\bar{F}(x)=\gamma(\bar{G}(x), c)
$$

Where $\bar{G}(x)=1-G(x)$. Aly and Benkherouf (2011) discoursed several properties of (6) and also provided the basis for the generalization of the Marshall-Olkin class considering the $p g f$ of the Harris distribution (Harris, 1948) for obtaining new distributions. This $p g f$ is given by

$$
\gamma(s, c, v)=\left(\frac{c s^{v}}{1-\bar{c} s^{v}}\right)^{\frac{1}{v}}, \quad v>0
$$

Although, Aly and Benkherouf (2011) considered $c>0$, Harris (1948) restricted to an open interval $(0,1)$. This constraint is a result of the fact that Harris distribution arises from a branching process for which each node may originate $v$ new nodes with probability proportional to $-\log (c)$. Putting (7) in (6) we obtain

$$
\bar{G}(x)=\left(\frac{c \bar{F}(x)^{v}}{1-\bar{c} \bar{F}(x)^{v}}\right)^{\frac{1}{v}}, \quad v>0
$$

The $p d f$ corresponding to (8) is given by

$$
g(x)=\frac{c^{\frac{1}{v}} f(x)}{\left[1-\bar{c} \bar{F}(x)^{v}\right]^{1+\frac{1}{v}}}, \quad v>0
$$

The equation (9) above reduces to the one proposed and studied by Marshall and Olkin when $v=1$.

The chief motivation of this study is based on the advantages of the generalized distribution with respect to having a hazard function that exhibits decreasing, increasing and bathtub shapes as well as the flexibility gain in compounding Harris distribution and Power Lomax distributions in modeling real life data. We develop and study a new distribution called the Harris Power Lomax distribution which possesses these properties with a wide range of applications.

The paper is organized as follows. In section 2, we examine the analytical shapes of the probability, survival and hazard functions under investigation and also the quantile function. Several properties of the proposed distribution are considered in section 3, majorly we obtain an expression for the moments (ordinary and incomplete moments), moment generating function, inequality measures (Bonferroni and Lorenz curves), stress-strength parameter, renyl entropy, and order statistics. In section 4, we evaluate the performance of the MLEs using simulation and two applications of HPL distribution to the Air craft windshield data and the Glass fibre data are carried out to examine its modeling potential. We provide the concluding remarks in section 5.

\section{Harris Power Lomax Distribution}

In this sub-section, we present the HPL distribution and derive some of its properties which include cdf, pdf, expansions of the density, hazard function, reversed hazard function, quantile function and sub-models. 
Consider $F(x)$ to be any baseline cdf in the interval $(0,1)$, by putting (4) in (8), we obtain the cdf of $H P L$ distribution given by

$$
G(x)=1-\left(\frac{c\left(1+\frac{x^{b}}{a}\right)^{-k v}}{1-\bar{c}\left(1+\frac{x^{b}}{a}\right)^{-k v}}\right)^{\frac{1}{v}}, \quad v>0
$$

The $p d f$ corresponding to (10) is given by

$$
g(x)=\frac{b c^{\frac{1}{v}} k x^{b-1}\left(1+\frac{x^{b}}{a}\right)^{-(k+1)}}{a\left[1-\bar{c}\left(1+\frac{x^{b}}{a}\right)^{-k v}\right]^{\left(1+\frac{1}{v}\right)}}, \quad v>0
$$

With $a, b, c, k, v>0$. Thus, $b, c, k$ and $v$ are the shape parameters and $a$ is a scale parameter. Plots of the pdf of $H P L$ distribution for various values of $a, b, c$ and $v$ are given in figures (1) and (2). The plots show that the $H L P$ is right (positive) skewed and can be decreasing ( $\mathrm{L}$ shape).

Graph of density function of HPL distribution, $v=0.5, c=0.5$

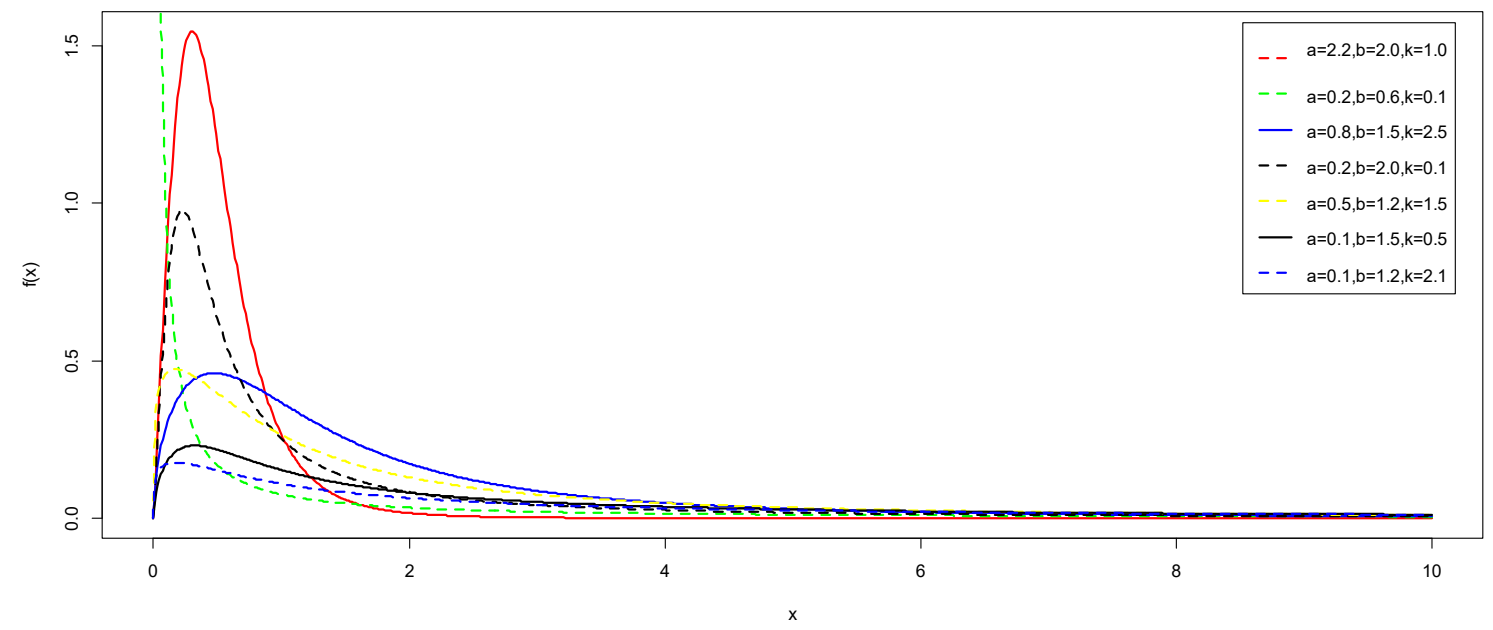

Figure 1. Plot of the PDF for different values of $a, c$ and $k$

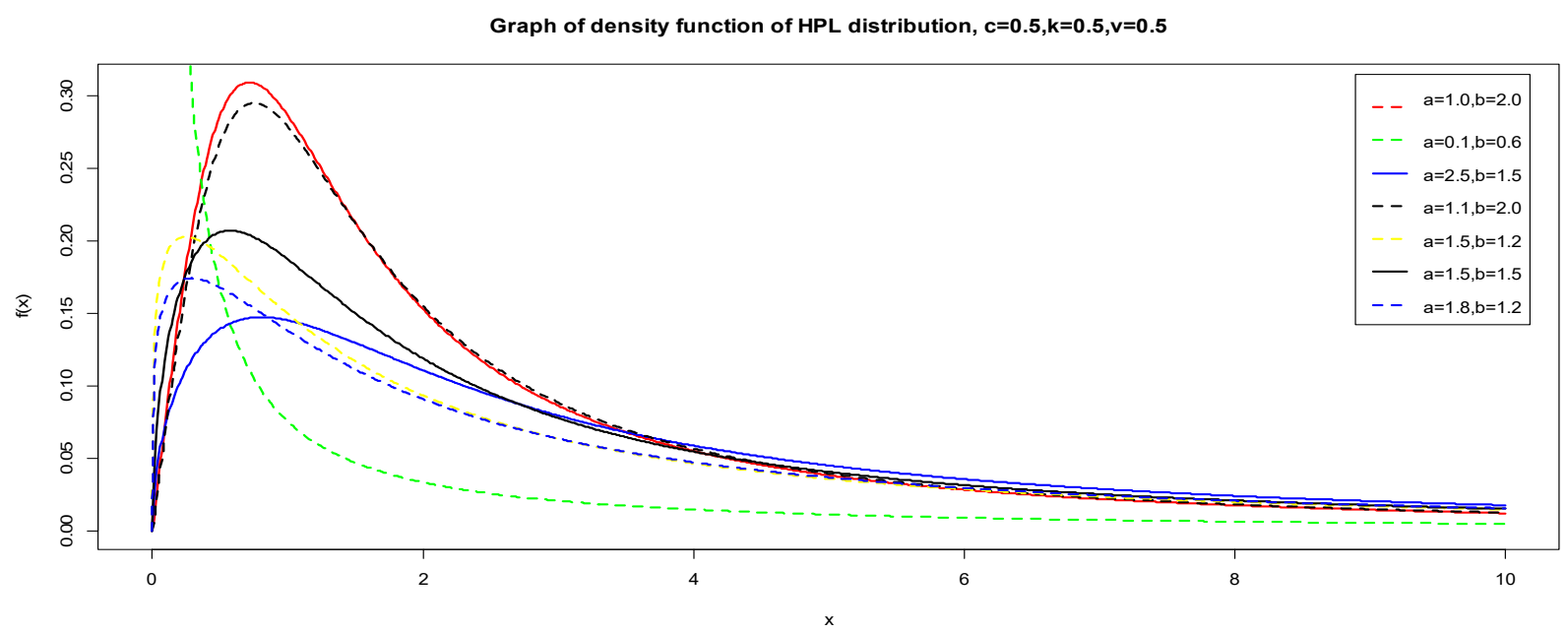

Figure 2. Plot of the pdf of HPL distribution for different values of $a$, and $b$ keeping $c, k$ and $v$ constant 


\subsection{Expansion of the Density Function}

The expansion of the pdf of HPL distribution is described in the sub-section. For $z>0$ a real non-integer, we use the series representation given by

$$
\{1-m\}^{-z}=\sum_{i=0}^{\infty}\left(\begin{array}{c}
z+i-1 \\
i
\end{array}\right) m^{i}
$$

We can rewrite the density of the HPL distribution as

$$
g(x)=\frac{b c^{\frac{1}{v}} k}{a} x^{b-1}\left(1+\frac{x^{b}}{a}\right)^{-(k+1)}\left[1-\bar{c}\left(1+\frac{x^{b}}{a}\right)^{-k v}\right]^{-\left(1+\frac{1}{v}\right)}
$$

Using (12) in (13), we observe that

$$
\left[1-\bar{c}\left(1+\frac{x^{b}}{a}\right)^{-k v}\right]^{-\left(1+\frac{1}{v}\right)}=\sum_{i=0}^{\infty}\left(\begin{array}{c}
\frac{1}{v}+i \\
i
\end{array}\right) \bar{c}^{i}\left(1+\frac{x^{b}}{a}\right)^{-k v i}
$$

Then (12) can be re-written in reduce form as

$$
g(x)=\sum_{i=0}^{\infty} \frac{b c^{\frac{1}{v}}}{a(v i+1)}\left(\begin{array}{c}
v^{-1}+i \\
i
\end{array}\right) \bar{c}^{i} k(v i+1) x^{b-1}\left(1+\frac{x^{b}}{a}\right)^{-[k(v i+1)+1]}
$$

Consequently,

$$
g(x)=\sum_{i=0}^{\infty} A_{i} f_{(v i+1) k}(x)
$$

Where

$$
A_{i}=\left\{\begin{array}{cl}
\frac{c^{\frac{1}{v}} \bar{c}^{i}}{1+i v}\left(\begin{array}{c}
i+v^{-1} \\
i
\end{array}\right) \quad & \text { for } c<1 \\
\frac{\tau(-1)^{i}}{(v i+1)}\left[\sum_{j=i}^{\infty} \bar{\tau}^{j}\left(\begin{array}{l}
j \\
i
\end{array}\right)\left(\begin{array}{c}
j+v^{-1} \\
j
\end{array}\right)\right], \quad \text { for } c \geq 1
\end{array}\right.
$$

Here, $\tau=c^{-1}$ and $f_{(v i+1) k}(x)$ denote the exponentiated Power Lomax distribution with parameters with scale parameter $k(v i+1)$. This suggests that the $H P L$ distribution can be written as a linear combination of Power Lomax density functions. Hence mathematical properties of the HPL distribution can be obtained from those of the $P L$ properties.

\subsection{Survival and Hazard Rate Functions}

The hazard function for the HPL distribution will be presented in this sub-section. The Survival and hazard function of the $H P L$ are respectively given by

$$
S_{H P L}(x)=1-G_{H P L}(x ; a, b, c, k, v)=\left(\frac{c\left(1+\frac{x^{b}}{a}\right)^{-k v}}{1-\bar{c}\left(1+\frac{x^{b}}{a}\right)^{-k v}}\right)^{\frac{1}{v}}
$$

for $x>0, a>0, b>0>c>0>, v>0$. The graph of the Survival function is given in figure 3 for various values of the parameters $a, b, c, k$ and $v$ 


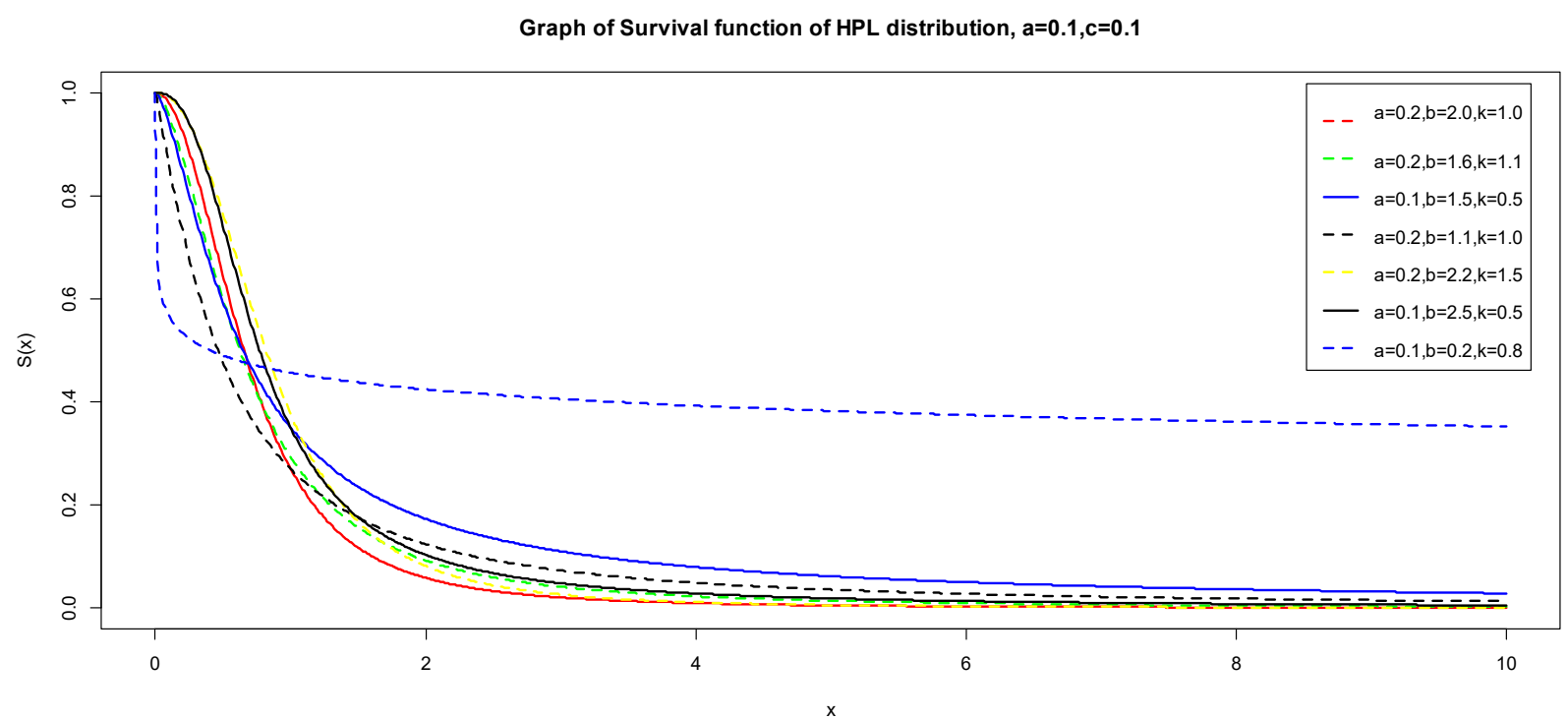

Figure 3. Graph of the survival probability of $H P L$ distribution

and

$$
\begin{array}{r}
h_{H P L}(x)=\frac{f_{H P L}(x ; a, b, c, k, v)}{S_{H P L}(x ; a, b, c, k, v)} \\
=\frac{\frac{b k}{a} x^{b-1}\left[1-\bar{c}\left(1+\frac{x^{b}}{a}\right)^{-k v}\right]^{-\left(1+k+\frac{1}{v}\right)}}{\left(1+\frac{x^{b}}{a}\right)}
\end{array}
$$

for $x>0, a>0, b>0>c>0>, v>0$. The graph of the hazard function are given in figure 4 and 5 for various values of the parameters $a, b, c, k$ and $v$. This graph shows that the $H P L$ distribution is

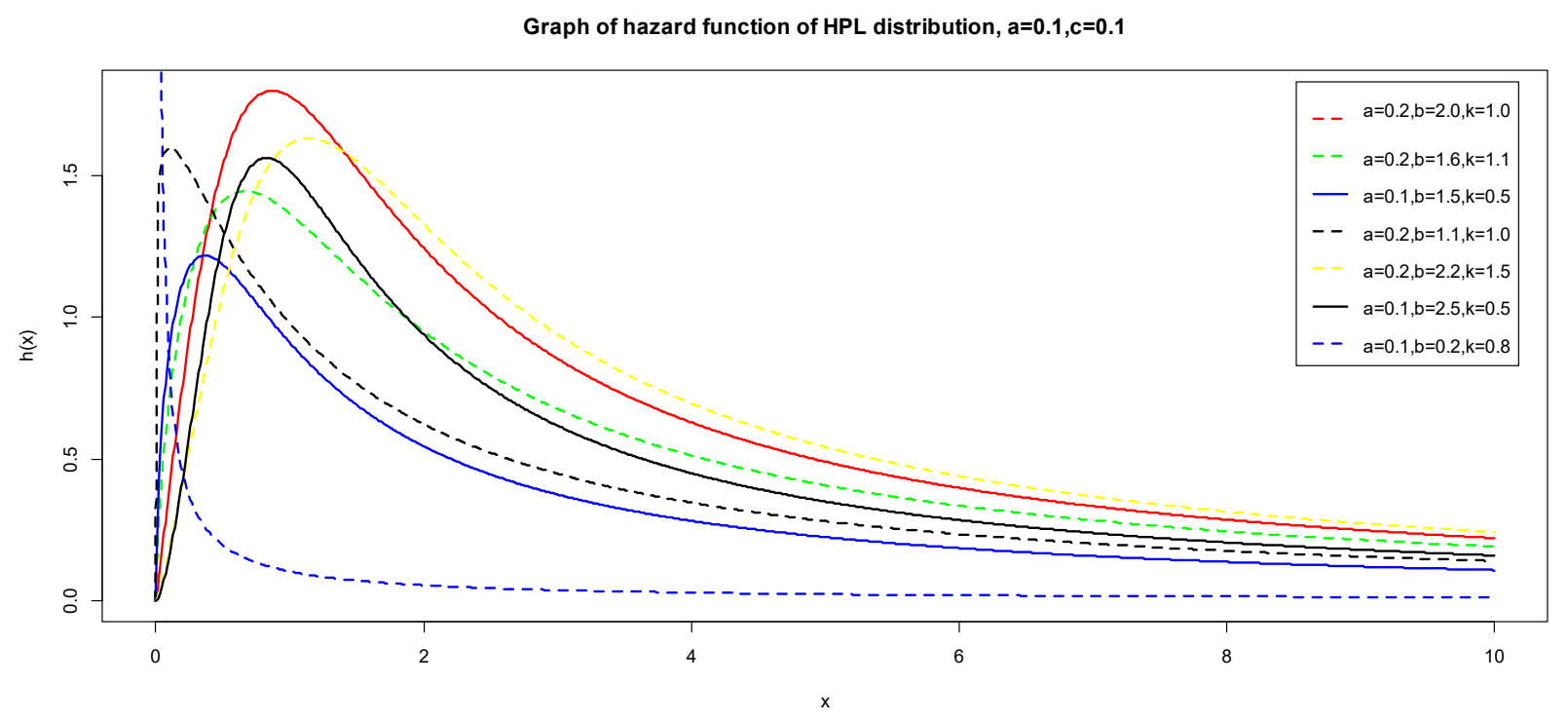

Figure 4. Graph of the hazard function of HPL distribution 


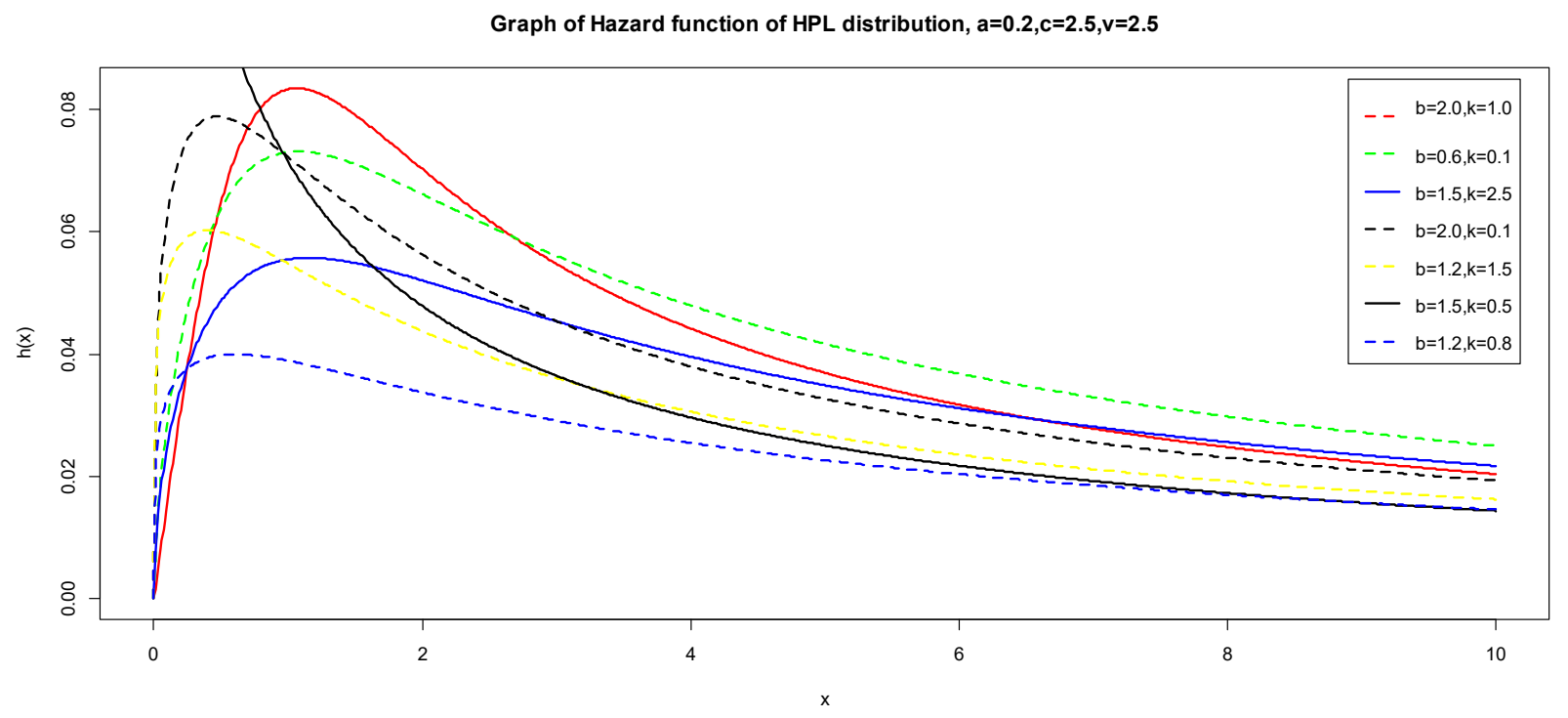

Figure 5. Graph of the hazard function of HPL distribution

\subsection{Quantile Function and Applications}

The quantile function of a distribution is an important tool in describing some important properties of the distribution. In this section, we present the quantile function of the HPL distribution, as well as some related properties, applications, and functions.

The quantile function of the $H$ distribution is obtained by solving the equation $Q(u ; a, b, c, k, v)=F^{-1}(u ; a, b, c, k, v)$, where $0<u<1$. After some algebraic manipulations, we obtain.

$$
Q(u ; a, b, c, k, v)=\left[a\left\{\left[\frac{(1-u)^{v}}{c+(1-c)(1-u)^{v}}\right]^{-\frac{1}{k v}}-1\right\}\right]^{\frac{1}{b}}, u \in(0,1) .
$$

In particular, we can obtain the lower quartile $\left(q_{1}\right)$, middle quartile or median $\left(q_{2}\right)$, and the Upper quartile $\left(q_{3}\right)$ of $H P L$ distribution by respectively taken $u=0.25,0.5$ and 0.75 . The expression for the distribution of quartiles is given below as

$$
\begin{gathered}
\left(q_{1}\right)=Q(0.25 ; a, b, c, k, v)=\left[a\left\{\left[\frac{1}{c+(1-c)(0.75)^{v}}\right]^{-\frac{1}{k v}}-1\right\}\right]^{\frac{1}{b}}, \\
\left(q_{2}\right)=Q(0.5 ; a, b, c, k, v)=\left[a\left\{\left[\frac{1}{c+(1-c)(0.5)^{v}}\right]^{-\frac{1}{k v}}-1\right\}\right]^{\frac{1}{b}},
\end{gathered}
$$

and

$$
\left(q_{3}\right)=Q(0.75 ; a, b, c, k, v)=\left[a\left\{\left[\frac{1}{c+(1-c)(0.25)^{v}}\right]^{-\frac{1}{k v}}-1\right\}\right]^{\frac{1}{b}}
$$

The mode of $H P L$ distribution can be obtained by solving the following equation $\frac{\delta}{\delta x}(\log [g(x ; a, b, c, k, v)])=0$. 


$$
\frac{\delta}{\delta x}(\log [g(x)])=\frac{b-1}{x}-\frac{(k+1)}{a}-(k+1) \frac{x^{b-1}}{a\left(1+\frac{x^{b}}{a}\right)}-\left(1+\frac{1}{v}\right) \frac{b \bar{c} k v x^{b-1}\left(1+\frac{x^{b}}{a}\right)^{-k v-1}}{a\left[1-\bar{c}\left(1+\frac{x^{b}}{a}\right)^{-k v}\right]}
$$

\subsection{Skewness and Kurtosis Based on Quantile Function}

The moments of distribution offers an empirical approach to measure the skewness and kurtosis of a distribution. However, in some instances the moments of a distribution do not always exist. This is a situation with heavy tail distribution such as the Power Lomax and Lomax distribution. In particular, to measure skewness of $H P L$ distribution, we consider Galton Skewness coefficient defined by

$$
G S=\frac{Q(3 / 4 ; a, b, c, k, v)+Q(1 / 4 ; a, b, c, k, v)-2 Q(2 / 4 ; a, b, c, k, v)}{Q(3 / 4 ; a, b, c, k, v)-Q(1 / 4 ; a, b, c, k, v)}
$$

To evaluate the value of kurtosis, we consider the Moor's kurtosis coefficient defined by

$$
K=\frac{Q(7 / 8 ; a, b, c, k, v)-Q(5 / 8 ; a, b, c, k, v)+Q(3 / 8 ; a, b, c, k, v)-Q(1 / 8 ; a, b, c, k, v)}{Q(6 / 8 ; a, b, c, k, v)-Q(2 / 8 ; a, b, c, k, v)}
$$

The sign on the value of $S$ provides information on the direction of the skewness of a distribution. In such that, when $S>0$, the distribution is right skewed, $S=0$ for symmetric distribution and $S<0$ for left skewed distribution. The value $K$ measures the tail heaviness of the distribution. In general, the bigger the value of $K$ is, the heavier is the tail of the distribution.

Table 1 drawn below presents the numerical values of the lower quartiles $\left(q_{1}\right)$, Median $\left(q_{2}\right)$, the upper quartile $\left(q_{3}\right)$, Galton Skewness $(G S)$ coefficient, and Moor's Coefficient $(K)$ of kurtosis of HPL distribution for fixed values of the parameters $(a=1.5, b=1.5, k=1.6)$ and varying the parameters $(c$ and $v)$

Table 1. Lower, Middle, Upper quartiles, Skewness and Kurtosis of HPL distribution

\begin{tabular}{|c|c|c|c|c|c|}
\hline$c, v$ & $q_{1}$ & $q_{2}$ & $q_{3}$ & $S$ & $K$ \\
\hline $0.1,0.2$ & 0.3838 & 0.5637 & 0.7898 & 0.1138 & 1.2017 \\
\hline $0.2,0.4$ & 0.5169 & 0.7842 & 1.1741 & 0.1866 & 1.3386 \\
\hline $0.5,0.7$ & 0.7752 & 1.2539 & 2.1522 & 0.3047 & 1.4073 \\
\hline $0.7,1.2$ & 0.9095 & 1.5317 & 2.8394 & 0.3552 & 1.7547 \\
\hline $0.8,2.0$ & 0.9729 & 1.6693 & 3.1777 & 0.3683 & 1.7725 \\
\hline $1.0,2.5$ & 1.0636 & 1.8251 & 3.4744 & 0.3683 & 1.7724 \\
\hline
\end{tabular}

$\checkmark$ From Table 1 we can conclude that for a fixed value of $a, b$, and $k$ and increasing the values of $c$ and $v$, we observed that the values of lower quartile, middle quartile, upper quartiles, skewness and kurtosis increases and the HPL distribution is positively skewed and mesokurtic.

\section{Moments of HPLDistribution}

Moments plays an important role in statistical analysis and applications. The most important features of a distribution such as measure of central tendency, skewness, kurtosis, dispersion can be studied using moments.

Proposition 3.0. The $r^{\text {th }}$ moment of a random variable $X$ following the $H P L$ distribution, denoted by $\mu_{r}^{\prime}$ is

$$
E\left(x^{r}\right)=\sum_{i=0}^{\infty} \frac{a^{\frac{r+b}{b}} c^{\frac{1}{v}}}{a(v i+1)}\left(\begin{array}{c}
v^{-1}+i \\
i
\end{array}\right) \bar{c}^{i} k(v i+1) B\left[\frac{r}{b}+1, k(v i+1)-\frac{r}{b}\right]
$$

Proof: By definition, the $r^{\text {th }}$ moment is given by

$$
\mu_{r}^{\prime}=E\left(x^{r}\right)=\int_{-\infty}^{\infty} x^{r} g(x) d x
$$




$$
\begin{gathered}
=\int_{0}^{\infty} x^{r \frac{b c c^{\frac{1}{v}} k}{a}} x^{b-1}\left(1+\frac{x^{b}}{a}\right)^{-(k+1)}\left[1-\bar{c}\left(1+\frac{x^{b}}{a}\right)^{-k v}\right]^{-\left(\frac{v+1}{v}\right)} d x \\
=\sum_{i=0}^{\infty} \frac{b c^{\frac{1}{v}}}{a(v i+1)}\left(\begin{array}{c}
v^{-1}+i \\
i
\end{array}\right) \bar{c}^{i} k(v i+1) \int_{-\infty}^{\infty} x^{r+b-1}\left(1+\frac{x^{b}}{a}\right)^{-[k(v i+1)+1]} d x
\end{gathered}
$$

In order to obtain an expression for the moments, we consider the following lemma:

$$
W(x, a, b, k(v i+1))=\int_{0}^{\infty} x^{r+b-1}\left(1+\frac{x^{b}}{a}\right)^{-[k(v i+1)+1]} d x
$$

Now, by applying change of variables, $y=\frac{x^{b}}{a}, x=(a y)^{\frac{1}{b}}, d x=\frac{1}{b} a^{\frac{1}{b}} y^{\frac{1}{b}-1}$

$$
W(x, a, b, k(v i+1))=\frac{a^{\frac{r+b}{b}}}{b} \int_{-\infty}^{\infty} y^{\frac{r}{b}}(1+y)^{-[k(v i+1)+1]} d y
$$

Also taking $y=\frac{u}{1-u}, 1+y=(1-u)^{-1}, d y=\frac{1}{(1-u)}+\frac{u}{(1-u)^{2}}$, we have

$$
W(x, a, b, k(v i+1))=\frac{a^{\frac{r+b}{b}}}{b} \int_{0}^{\infty} u^{\frac{r}{b}}(1-u)^{k(v i+1)-\frac{r}{b}-1} d u
$$

finally

$$
W(x, a, b, k(v i+1))=\frac{a^{\frac{r+b}{b}}}{b} B\left[\frac{r}{b}+1, k(v i+1)-\frac{r}{b}\right]
$$

Where $B(m, n)$ denotes the standard beta function defined by $B(m, n)=\int_{0}^{1} z^{m-1}(1-z)^{y-1} d z$

Consequently, the expression for the $r^{\text {th }}$ moment of HPL distribution is given by

$$
E\left(x^{r}\right)=\sum_{i=0}^{\infty} \frac{a^{\frac{r+b}{b}} c^{\frac{1}{v}}}{a(v i+1)}\left(\begin{array}{c}
v^{-1}+i \\
i
\end{array}\right) \bar{c}^{i} k(v i+1) B\left[\frac{r}{b}+1, k(v i+1)-\frac{r}{b}\right]
$$

By taking $r=1$, we obtain the mean of $X$, i.e., $\mu=\mu_{1}^{\prime}$. The variance of $X$ is obtained by $\delta^{2}=E\left\{(X-\mu)^{2}\right\}=$ $\mu_{1}^{\prime}-\mu^{2}$. In addition, one can determine the $r^{\text {th }}$ central moment and $r^{\text {th }}$ cumulant of $X$ defined by, respectively,

$$
\mu_{r}=E\left\{(X-\mu)^{r}\right\}=\sum_{k=0}^{r}\left(\begin{array}{l}
r \\
k
\end{array}\right) \mu_{r-k}^{\prime}(-1)^{k} \mu^{k}, \mathcal{K}_{r}=\mu_{r}^{\prime}-\sum_{k=1}^{r-1}\left(\begin{array}{l}
r-1 \\
k-1
\end{array}\right) \mathcal{K}_{k} \mu_{r-k}^{\prime}
$$

With $\mathcal{K}_{1}=\mu$. Measures of skewness and kurtosis and kurtosis can also be expressed in a similar manner.

\subsection{Incomplete Moments of HPL Distribution}

Proposition 3.1: Let $X$ be a random variable following the $H P L$ distribution and for any $t \geq 0$, let $X_{t}=X$ if $X \leq t$ and 0 otherwise. Then, the $r^{\text {th }}$ incomplete moment of $\mathrm{X}$ is given by

$$
E\left(X_{t}^{r}\right)=\sum_{i=0}^{\infty} \frac{a^{\frac{r+b}{b}} c^{\frac{1}{v}}}{a(v i+1)}\left(\begin{array}{c}
v^{-1}+i \\
i
\end{array}\right)(\bar{c})^{i} k(v i+1) B\left[\begin{array}{l}
r \\
b
\end{array}+1, k(v i+1)-\frac{r}{b}\right]
$$

Proof: By definition, an expression for the $r^{t h}$ incomplete moment of $\mathrm{X}$ is given by

$$
\mu_{r}^{\prime}(t)=E\left(X_{t}^{r}\right)=\int_{0}^{t} x^{r} g(x ; a, b, c, k \cdot v) d x
$$




$$
\begin{gathered}
=\sum_{i=0}^{\infty} \frac{b c^{\frac{1}{v}}}{a(v i+1)}\left(\begin{array}{c}
v^{-1}+i \\
i
\end{array}\right) \bar{c}^{i} k(v i+1) \int_{0}^{t} x^{r+b-1}\left(1+\frac{x^{b}}{a}\right)^{-[k(v i+1)+1]} d x \\
\varphi(x, a, b, k(v i+1))=\int_{0}^{t} x^{r+b-1}\left(1+\frac{x^{b}}{a}\right)^{-[k(v i+1)+1]} d x
\end{gathered}
$$

Now, by applying change of variables, $y=\frac{x^{b}}{a}, x=(a y)^{\frac{1}{b}}, d x=\frac{1}{b} a^{\frac{1}{a}} y^{\frac{1}{b}-1}$

$$
\varphi(x, a, b, k(v i+1))=\frac{a^{\frac{r+b}{b}}}{b} \int_{0}^{\frac{t^{b}}{a}} y^{\frac{r}{b}}(1+y)^{-[k(v i+1)+1]} d y
$$

Also taking $y=\frac{u}{1-u}, 1+y=(1-u)^{-1}, d y=\frac{1}{(1-u)}+\frac{u}{(1-u)^{2}}$, we have

$$
\varphi(t, a, b, k(v i+1))=\frac{a^{\frac{r+b}{b}}}{b} \int_{0}^{\frac{t^{b}}{\left(t^{b}+a\right)}} u^{\frac{r}{b}}(1-u)^{k(v i+1)-\frac{r}{b}-1} d y
$$

finally

$$
\varphi(t, a, b, k(v i+1))=\frac{a^{\frac{r+b}{b}}}{b} B\left[\frac{t^{b}}{a+t^{b}} ; \frac{r}{b}+1, k(v i+1)-\frac{r}{b}\right]
$$

where $B(x ; m, n)=\int_{0}^{x} z^{m-1}(1-z)^{n-1} d z$, is the beta function.

Consequently, the expression for the $r^{t h}$ incomplete moment of HPL distribution is given by

$$
E\left(X_{t}^{r}\right)=\sum_{i=0}^{\infty} \frac{a^{\frac{r+b}{b}} c^{\frac{1}{v}}}{a(v i+1)}\left(\begin{array}{c}
v^{-1}+i \\
i
\end{array}\right)(\bar{c})^{i} k(v i+1) B\left[\frac{t^{b}}{a+t^{b}} ; \frac{r}{b}+1, k(v i+1)-\frac{r}{b}\right]
$$

From the incomplete moment of $X$, other important quantities and functions of $X$ can be obtained. For instance, one can express the mean deviations of $X$ about the mean $(\mu)$ and the median $(M)$ respectively, by,

$$
\delta_{1}=E(|X-\mu|)=2 \mu F(\mu ; a, b, c, k, v)-2 \mu_{1}^{\prime}(\mu), \quad \delta_{2}=E(|X-M|)=\mu-2 \mu_{1}^{\prime}(M) .
$$

Other quantities can also be derived in similar manner. Other well-known functions defined with the r-incomplete moment include the $r^{\text {th }}$ lower and upper conditional moments of $X$ defined respectively, by,

And

$$
\mu_{r}^{\nabla}(t)=E\left(X^{r} / X \leq t\right)=\frac{1}{G(t ; a, b, c, k, v)} \mu_{r}^{\prime}(t), \quad t \geq 0
$$

$$
\mu_{r}^{\Delta}(t)=E\left(X^{r} / X>t\right)=\frac{1}{S(t ; a, b, c, k, v)}\left[\mu_{r}^{\prime}-\mu_{r}^{\prime}(t)\right], \quad t \geq 0 .
$$

\subsection{Moment Generating Function of HPL Distribution}

Proposition 3.2: if $X$ has the $\operatorname{HPL}(x ; v, w, \lambda)$ distribution, then the moment generating function of $\mathrm{X}$, say $M_{x}(t)$ is given as

$$
M_{x}(t)=\sum_{i=0}^{\infty} \sum_{r=0}^{\infty} \frac{t^{r}}{r !} \frac{a^{\frac{r+b}{b}} c^{\frac{1}{v}}}{a(v i+1)}\left(\begin{array}{c}
v^{-1}+i \\
i
\end{array}\right) \bar{c}^{i} k(v i+1) B\left[\frac{r}{b}+1, k(v i+1)-\frac{r}{b}\right]
$$

Proof: by definition, the moment generating function of a random variable $X$ is given by 


$$
M_{x}(t)=E\left(e^{t x}\right)=\int_{-\infty}^{\infty} e^{t x} g(x) d x
$$

Using Taylor series expansion,

$$
e^{t x}=\sum_{r=o}^{\infty} \frac{t^{r}}{r !} E\left(X^{r}\right)
$$

Therefore,

$$
M_{x}(t)=\sum_{r=o}^{\infty} \frac{t^{r}}{r !} E\left(X^{r}\right)
$$

Putting equation (23) in (28), we obtain an expression for the moment generating function of HPL distribution as

$$
M_{x}(t)=\sum_{i=0}^{\infty} \sum_{r=0}^{\infty} \frac{t^{r}}{r !} \frac{a^{\frac{r+b}{b}} c^{\frac{1}{v}}}{a(v i+1)}\left(\begin{array}{c}
v^{-1}+i \\
i
\end{array}\right) \bar{c}^{i} k(v i+1) B\left[\begin{array}{l}
r \\
b
\end{array}+1, k(v i+1)-\frac{r}{b}\right]
$$

where $B(x ; m, n)=\int_{0}^{x} z^{m-1}(1-z)^{n-1} d z$, is the beta function

\subsection{Inequality Measures}

The Bonferroni and Lorenz curves are widely used measures of income inequality of a given population and have various applications in, insurance, economics, medicine, and reliability.

Proposition 3.3: The Bonferroni curve for the HPL distribution is given by

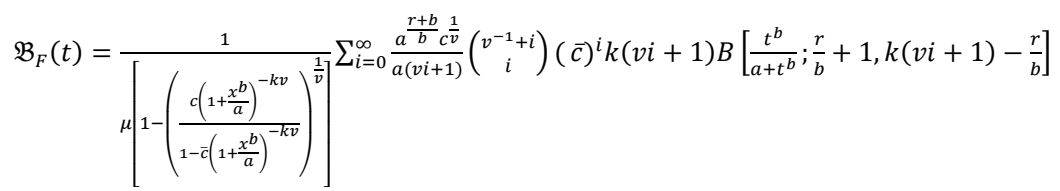

\section{Proof: By definition}

$$
\begin{gathered}
\mathfrak{B}_{F}(t)=\frac{1}{\mu G(t)} \int_{0}^{t} x^{r} g(x ; a, b, c, k, v) d x \\
=\frac{1}{\mu\left[1-\left(\frac{c\left(1+\frac{t^{b}}{a}\right)^{-k v}}{1-\bar{c}\left(1+\frac{t^{b}}{a}\right)^{-k v}}\right)^{\frac{1}{v}} \sum_{i=0}^{\infty} \frac{a^{\frac{r+b}{b}} c^{\frac{1}{v}}}{a(v i+1)}\left(\begin{array}{c}
v^{-1}+i \\
i
\end{array}\right)(\bar{c})^{i} k(v i+1) B\left[\frac{r}{b}+1, k(v i+1)-\frac{r}{b}\right],\right.}
\end{gathered}
$$

It should be noted that $\mu=\mu_{1}^{\prime}$ equal the first about the origin and is obtained by taking $r=1$ in equation (23).

Proposition 3.4: The Lorenz curve for the HPL distribution is given by

$$
L_{F}(t)=\frac{1}{\mu} \sum_{i=0}^{\infty} \frac{a^{\frac{r+b}{b}} c^{\frac{1}{v}}}{a(v i+1)}\left(\begin{array}{c}
v^{-1}+i \\
i
\end{array}\right)(\bar{c})^{i} k(v i+1) B\left[\frac{r}{b}+1, k(v i+1)-\frac{r}{b}\right], t>0, r<b
$$

Proof: By definition

$$
L_{F}(t)=\frac{1}{\mu} \int_{0}^{t} x^{r} g(x ; a, b, c, k, v) d x
$$




$$
=\frac{1}{\mu} \sum_{i=0}^{\infty} \frac{a^{\frac{r+b}{b}} c^{\frac{1}{v}}}{a(v i+1)}\left(\begin{array}{c}
v^{-1}+i \\
i
\end{array}\right)(\bar{c})^{i} k(v i+1) B\left[\frac{r}{b}+1, k(v i+1)-\frac{r}{b}\right], t>0, r<b
$$

\subsection{Stress-Strength Parameter}

Suppose we let $X_{1}$ and $X_{2}$ be two continuous and independent random variables, where $X_{1} \sim H P L\left(a_{1}, c_{1}, v_{1}\right)$ and $X_{2} \sim H P L\left(a_{2}, c_{2}, v_{2}\right)$, then the series strength parameter, say 2 , is defined as

$$
2=\int_{-\infty}^{\infty} g_{1}(x) G_{2}(x) d x
$$

Using the pdf and the cdf of HPL given in equation (10) and (11) in (31), we have

$$
\chi=\frac{b}{a} c_{1}^{1 / v_{1}} \bar{c}_{2}^{1 / v_{2}} k \sum_{i=0}^{\infty} \sum_{j=0}^{\infty} c_{1}^{i} \bar{c}_{2}^{j}\left(\begin{array}{c}
v_{1}^{-1}+i \\
i
\end{array}\right)\left(\begin{array}{c}
v_{2}^{-1}+j-1 \\
j
\end{array}\right) \int_{0}^{\infty} x^{b-1}\left(1+\frac{x^{b}}{a}\right)^{-\left[k\left(2+v_{1} i+v_{2} j\right)+1\right]} d x
$$

Now, by applying change of variables $x=(a y)^{1 / b}$ and $y=\frac{u}{1-u}$ twice in a row, we get

$$
\mathcal{2}=a^{b-1} c_{1}^{1 / v_{1}} \bar{c}_{2}^{1 / v_{2}} k \sum_{i=0}^{\infty} \sum_{j=0}^{\infty} c_{1}^{i} \bar{c}_{2}^{j}\left(\begin{array}{c}
v_{1}^{-1}+i \\
i
\end{array}\right)\left(\begin{array}{c}
v_{2}^{-1}+j-1 \\
j
\end{array}\right) B\left[1, k\left(2+v_{1} i+v_{2} j\right)\right]
$$

\subsection{Renyi Entropy of HPL Distribution}

The Renyi entropy can be used to measures the uncertainty in a distribution as defined by Renyi (1961). The larger the value of entropy obtains the greater the level of uncertainty inherent in the distribution.

Proposition 3.5: If the random variable $X$ has a HPL distribution, the Renyi entropy of $\mathrm{X}$ is given by

$$
I_{z}(x)=(1-z)^{-1} \log \left[Q_{h}^{w} B\left[(z(b-1)+1) /{ }_{b}, z k+k j v+(z-1) / b\right]\right]
$$

Proof: By definition

$$
I_{z}(x)=(1-z)^{-1} \log \left[\int_{-\infty}^{+\infty} g^{z}(x) d x\right] d x, \quad z>0, z \neq 0
$$

Putting (13) in (32), we have

$$
I_{z}(x)=(1-z)^{-1} \log \left[\int_{-\infty}^{+\infty}\left\{\frac{b c^{\frac{1}{v}} k}{a} x^{b-1}\left(1+\frac{x^{b}}{a}\right)^{-(k+1)}\left[1-\bar{c}\left(1+\frac{x^{b}}{a}\right)^{-k v}\right]^{-\left(\frac{v+1}{v}\right)}\right\}^{z} d x\right]
$$

Since

$$
\left[1-\bar{c}\left(1+\frac{x^{b}}{a}\right)^{-k v}\right]^{-z\left(1+\frac{1}{v}\right)}=\sum_{j=0}^{\infty}\left(\begin{array}{c}
z(1+1 / v)+j-1 \\
j
\end{array}\right)(\bar{c})^{j}\left(1+\frac{x^{b}}{a}\right)^{-k v j}
$$

Therefore,

$$
I_{z}(x)=(1-z)^{-1} \log \left[\left(\frac{b c^{\frac{1}{v}} k}{a}\right)^{z} \int_{-\infty}^{+\infty} x^{z(b-1)}\left(1+\frac{x^{b}}{a}\right)^{-\{z(k+1)+k v j\}} d x\right]
$$

Also by making twice substitution, $y=\frac{x^{b}}{a}, y=\frac{u}{1-u}$, then we have 


$$
\int_{-\infty}^{+\infty} x^{z(b-1)}\left(1+\frac{x^{b}}{a}\right)^{-\{z(k+1)+k v j\}} d x=B\left[(z(b-1)+1) /{ }_{b}, z k+k j v+(z-1) / b\right]
$$

Finally, we have

$$
I_{z}(x)=(1-z)^{-1} \log \left[Q_{h}^{w} B[(z(b-1)+1) / b, z k+k j v+(z-1) / b]\right]
$$

where

$$
Q_{h}^{w}=b^{z-1} a^{\frac{1-z}{b}}(c)^{\frac{z}{v}} \sum_{j=0}^{\infty}\left(\begin{array}{c}
z(1+1 / v)+j-1 \\
j
\end{array}\right)(\bar{c})^{j}
$$

\subsection{Order Statistics of HPL Distribution}

Let $X$ be a random variable that follow the HPL distribution and, for a random sample of size $n$ from $X$, say $x_{1}, x_{2} \ldots, x_{n}$ be the $k^{t h}$ order statistics such that $x_{1: n} \leq x_{2: n} \leq \ldots \leq x_{n: n}$, where $x_{m: n} \in\left\{x_{1}, x_{2}, \ldots, x_{n}\right\}$ for $m=1,2, \ldots, n$. In real life testing the study of order statistics is of importance since they found applications in many systems, mainly those that compose of several components that can fail independently of each other. An expression for the $k^{\text {th }}$ order statistics is given by

$$
f_{i: n}(x ; a, b, c, k, v)=\zeta^{n, i} G(x ; a, b, c, k, v)^{i-1}[1-G(x ; a, b, c, k, v)]^{n-i} g(x ; a ; b ; c ; k ; v)
$$

Where

$$
\zeta^{n, i}=\frac{n !}{(i-1) !(n-i) !}
$$

Applying binomial formula given in (12) in (34), we have

$$
f_{i: n}(x ; a, b, c, k, v)=\zeta^{n, i} \sum_{j=0}^{n-i}(-1)^{j}\left(\begin{array}{c}
n-i \\
j
\end{array}\right)[G(x ; a, b, c, k, v)]^{i+j-1} g(x ; a, b, c, k, v)
$$

Then putting equation (10) and (13) in (35), we have

$$
\begin{aligned}
f_{i: n}(x ; a, b, c, k, v)= & \frac{b c^{\frac{1}{v}} k}{a} \zeta^{n, i} \sum_{j=0}^{n-i}(-1)^{j}\left(\begin{array}{c}
n-i \\
j
\end{array}\right)\left[1-\left(\frac{c\left(1+\frac{x^{b}}{a}\right)^{-k v}}{1-\bar{c}\left(1+\frac{x^{b}}{a}\right)^{-k v}}\right)^{\frac{1}{v}}\right]^{i+j-1} \\
& \times x^{b-1}\left(1+\frac{x^{b}}{a}\right)^{-(k+1)}\left[1-\bar{c}\left(1+\frac{x^{b}}{a}\right)^{-k v}\right]^{-\left(\frac{v+1}{v}\right)}
\end{aligned}
$$

By further algebraic manipulation, we obtain

$$
\begin{aligned}
f_{i: n}(x ; a, b, c, k, v)= & \frac{b k}{a} \zeta^{n, i} \sum_{j=0}^{n-i} \sum_{l=0}^{i+j-1} \sum_{m=0}^{\infty}(-1)^{j+l}\left(\begin{array}{c}
n-i \\
j
\end{array}\right)\left(\begin{array}{c}
i+j-1 \\
l
\end{array}\right)\left(\begin{array}{c}
\frac{v+l+1}{v}+m-1 \\
m
\end{array}\right) c^{\frac{l+1}{v}} \\
& \times(\bar{c})^{m} x^{b-1}\left(1+\frac{x^{b}}{a}\right)^{-[k(v m+l+1)+1]}
\end{aligned}
$$

By taking $n=1$, we obtain an expression for the first order statistics of $H P L$ distribution and taking $n=j$, we obtain an expression for the $n^{\text {th }}$ or the largest order statistics of $H P L$ distribution.

\section{Maximum Likelihood Estimation Method}

The maximum likelihood estimation is used to determine the parameters that maximize the likelihood function of the sample data. Suppose we take a random sample $x_{1}, \ldots, x_{n}$ from the $H L P$ distribution, the corresponding likelihood function is given by 


$$
\begin{aligned}
& L(a, b, c, k, v)=\prod_{i=1}^{n} g(x ; a, b, c, k, v) \\
& =\prod_{i=1}^{n}\left\{\frac{b c^{\frac{1}{v}} k}{a} x^{b-1}\left(1+\frac{x^{b}}{a}\right)^{-(k+1)}\left[1-\bar{c}\left(1+\frac{x^{b}}{a}\right)^{-k v}\right]^{-\left(1+\frac{1}{v}\right)}\right\}
\end{aligned}
$$

The MLEs of $a, b, c, k$, and $v$ are given by $\widehat{a}, \hat{b}, \widehat{c}, \widehat{k}$, and $\hat{v}$, respectively. The log-likelihood function is given by

$$
\begin{aligned}
l= & n\left[v^{-1} \log c\right]+n \log \left(\frac{b k}{a}\right)+(b-1) \sum_{i=0}^{n} \log \left(x_{i}\right)-(k+1) \sum_{i=0}^{n} \log \left(1+\frac{x_{i}^{b}}{a}\right) \\
& -\left(1+\frac{1}{v}\right) \sum_{i=0}^{n} \log \left[1-(1-c)\left(1+\frac{x_{i}^{b}}{a}\right)^{-k v}\right]
\end{aligned}
$$

Thus, $\widehat{a}, \widehat{b}, \widehat{c}, \widehat{k}$, and $\hat{v}$ are the simultaneous solutions of the following non-linear equations: $\frac{\partial l}{\partial a}=0, \frac{\partial l}{\partial b}=0, \frac{\partial l}{\partial c}=0, \frac{\partial l}{\partial k}=$ 0 , and $\frac{\partial l}{\partial v}=0$. Here, we obtained the partial derivatives that maximize the log-likehood function as,

$$
\begin{aligned}
& \frac{\partial l}{\partial a}=-\frac{n}{a}+(k+1) \sum_{i=0}^{n} \log \left[\frac{x_{i}^{b}}{a^{2}\left(1+\frac{x_{i}^{b}}{a}\right)}\right]+\left(1+\frac{1}{v}\right) \sum_{i=0}^{n}\left[\frac{(1-c) v k\left(1+\frac{x_{i}^{b}}{a}\right)}{a^{2}\left\{1-(1-c)\left(1+\frac{x_{i}^{b}}{a}\right)^{k v}\right\}}\right] \\
& \frac{\partial l}{\partial b}=\frac{n}{b}+\sum_{i=0}^{n} \log \left(x_{i}\right)+(k+1) \sum_{i=0}^{n} \log \frac{x_{i}^{b} \log x_{i}}{a\left(1+\frac{x_{i}^{b}}{a}\right)} \\
& -\left(1+\frac{1}{v}\right) \sum_{i=0}^{n} \frac{(1-c) v k\left[x_{i}^{b} \log \left(x_{i}\right)\right]}{\left(1+\frac{x_{i}^{b}}{a}\right)\left[1-(1-c)\left(\left(1+\frac{x_{i}^{b}}{a}\right)^{-k v}\right]\right.} \\
& \frac{\partial l}{\partial c}=\frac{n}{v c}-\left(1+\frac{1}{v}\right) \sum_{i=0}^{n}\left\{\frac{\left(1+\frac{x_{i}^{b}}{a}\right)^{-k v}}{\left[1-\bar{c}\left(1+\frac{x_{i}^{b}}{a}\right)^{-k v}\right]}\right\} \\
& \frac{\partial l}{\partial k}=\frac{n}{k}-\sum_{i=0}^{n} \log \left(1+\frac{x_{i}^{b}}{a}\right)-\left(1+\frac{1}{v}\right) \sum_{i=0}^{n} \frac{v(1-c)\left(1+\frac{x_{i}^{b}}{a}\right)^{-k v} \log \left(1+\frac{x_{i}^{b}}{a}\right)}{\left[1-(1-c)\left(1+\frac{x_{i}^{b}}{a}\right)^{-k v}\right]} \\
& \frac{\partial l}{\partial v}=-\frac{n \log (c)}{v^{2}}+v^{-2} \sum_{i=0}^{n} \log \left[1-(1-c)\left(1+\frac{x_{i}^{b}}{a}\right)^{-k v}\right] \\
& -\left(1+\frac{1}{v}\right) \sum_{i=0}^{n} \frac{(1-c) k v x_{i}^{b} \log \left(x_{i}\right)}{a\left(1+\frac{x_{i}^{b}}{a}\right)\left[1-(1-c)\left(1+\frac{x_{i}^{b}}{a}\right)^{-k v}\right]}
\end{aligned}
$$

The analytical solution to the system of nonlinear equations presented in (40), (41), (42), (43), and (44) does not exist. Therefore, a numerical method such as OX program, nlminb function in R program etc. is needed to obtain the solution. The approximate confidence interval of the parameters $\underline{\theta}=(a, b, c, k, v)$ can be obtained based on the asymptotic distribution of the MLE's of $\underline{\theta}$. Considering a large sample under appropriate regularity conditions, the MLEs for the parameters $\hat{\theta}=(\hat{a}, \hat{b}, \hat{c}, \hat{k}, \hat{v})$ have approximate multivariate normal distribution and asymptotic variance-covariance matrix which can be used to approximate the inverse of Fisher information matrix. Then $100(1-\Upsilon / 2) \%$ approximate confidence interval of the parameters $\underline{\theta}=(a, b, c, k, v)$ are: 


$$
\begin{gathered}
\hat{a} \pm z_{\Upsilon / 2} \sqrt{\operatorname{var}(\hat{a})}, \hat{b} \pm z_{\Upsilon / 2} \sqrt{\operatorname{var}(\hat{b})}, \hat{c} \pm z_{\Upsilon / 2} \sqrt{\operatorname{var}(\hat{c})}, \hat{k} \pm z_{\Upsilon / 2} \sqrt{\operatorname{var}(\hat{k})} \text { and } \\
\hat{v} \pm z_{\Upsilon / 2} \sqrt{\operatorname{var}(\hat{v})}
\end{gathered}
$$

Where $z_{\Upsilon / 2}$ is the upper $Y^{\text {th }}$ percentile of the normal distribution.

\subsection{Simulation Results of HPL Distribution}

We carry out Monte Carlo simulations to show the asymptotic property of the MLEs for the HPL distribution. We calculate Absolute Biases $(A B)$, Standard Error $(S E)$, and mean squared errors (MSEs) of each parameter for different sample sizes. To obtain the results, the process is replicated $N=1,000$ times for $n=50,100,150,200$, and 250 for fixed choice of parameters $a=0.3, b=1.4, c=1.5, k=0.6$, and $v=1.5$. The estimates of the unknown parameters have been obtained by using BFGS method to minimize the total log-likelihood function. The estimated values of the parameters $a, b, c, k$, and $v$ with their corresponding Standard Error $(S E)$, Absolute Bias $(A B)$ and Mean Square Error (MSE) are displayed in Table 2. The $A B$ and $M S E$ for an estimator $\hat{\theta}$ are defined by

$$
A B(\hat{\theta})=\left|\frac{\sum_{i}^{N} \hat{\theta}}{N}-\theta\right|, \quad M S E(\hat{\theta})=\frac{\sum_{i}^{N}(\hat{\theta}-\theta)^{2}}{N}
$$

It can be noticed from the simulated results in Table $2 M S E$ of the estimators of all parameters decrease with the

\begin{tabular}{|c|c|c|c|c|c|}
\hline Parameter & $\begin{array}{c}\text { Sample size } \\
(n)\end{array}$ & $\begin{array}{l}\text { Average } \\
\text { estimate }\end{array}$ & $A B$ & $S E$ & $M S E$ \\
\hline \multirow{5}{*}{$v=1.5$} & 50 & 0.6233 & 0.8767 & 1.8117 & 4.0509 \\
\hline & 100 & 0.9335 & 0.5665 & 1.9363 & 4.0702 \\
\hline & 150 & 0.9102 & 0.5898 & 1.8165 & 3.6475 \\
\hline & 200 & 0.8473 & 0.6527 & 0.9485 & 1.3257 \\
\hline & 250 & 1.0365 & 0.4635 & 0.9425 & 1.1031 \\
\hline \multirow{5}{*}{$k=0.6$} & 50 & 0.6994 & 0.0994 & 0.8847 & 0.7926 \\
\hline & 100 & 0.7265 & 0.1265 & 0.3399 & 0.1315 \\
\hline & 150 & 0.5262 & 0.0738 & 0.2378 & 0.0620 \\
\hline & 200 & 0.4925 & 0.1075 & 0.2020 & 0.0524 \\
\hline & 250 & 0.5480 & 0.0520 & 0.1258 & 0.0185 \\
\hline \multirow{5}{*}{$c=1.5$} & 50 & 0.3084 & 1.1916 & 1.3408 & 3.2177 \\
\hline & 100 & 0.4188 & 1.0812 & 1.9865 & 5.1152 \\
\hline & 150 & 0.3214 & 1.1786 & 1.0605 & 2.5138 \\
\hline & 200 & 0.2074 & 1.2926 & 0.4183 & 1.8458 \\
\hline & 250 & 0.3526 & 1.1474 & 0.3039 & 1.4089 \\
\hline \multirow{5}{*}{$b=1.4$} & 50 & 1.5089 & 0.1089 & 0.3248 & 0.1174 \\
\hline & 100 & 1.4097 & 0.0097 & 0.2350 & 0.0553 \\
\hline & 150 & 1.6032 & 0.2032 & 0.2232 & 0.0911 \\
\hline & 200 & 1.6179 & 0.2179 & 0.1762 & 0.0785 \\
\hline & 250 & 1.5129 & 0.1129 & 0.1675 & 0.0408 \\
\hline \multirow{5}{*}{$a=0.3$} & 50 & 1.6562 & 1.3562 & 7.0966 & 52.2010 \\
\hline & 100 & 1.4024 & 1.1024 & 6.5947 & 44.7054 \\
\hline & 150 & 1.1596 & 0.8596 & 3.9294 & 16.1791 \\
\hline & 200 & 1.6420 & 1.3420 & 3.3243 & 12.8519 \\
\hline & 250 & 1.5356 & 1.2356 & 3.0529 & 10.8469 \\
\hline
\end{tabular}
increasing sample size.

Table 2. Simulation Results for $H P L$ distribution

\subsection{Real Data Applications}

The following are the data sets which we have used in this study to validate the modeling capability of Harris Power Lomax distribution. Data set 1 set signifies the failure times of 84 aircraft windshield device which was studied by Murphy et al. (2004).

Data Set 1: $0.040,1.866,2.385,3.443,0.301,1.876,2.481,3.467,0.309,1.899,2.610,3.478,0.557,1.911,2.625,3.578$, $0.943,1.912,2.632,3.595, \quad 1.070, \quad 1.914, \quad 2.646, \quad 3.699, \quad 1.124, \quad 1.981, \quad 2.661,3.779,1.248, \quad 2.010, \quad 2.688$, $3.924, \quad 1.281, \quad 2.038, \quad 2.82,3, \quad 4.035, \quad 1.281,2.085, \quad 2.890, \quad 4.121, \quad 1.303,2.089, \quad 2.902, \quad 4.167, \quad 1.432$, 
$2.097, \quad 2.934, \quad 4.240, \quad 1.480, \quad 2.135, \quad 2.962, \quad 4.255, \quad 1.505, \quad 2.154, \quad 2.964,4.278, \quad 1.506, \quad 2.190, \quad 3.000$, $4.305, \quad 1.568, \quad 2.194, \quad 3.103, \quad 4.376, \quad 1.615, \quad 2.223, \quad 3.114, \quad 4.449, \quad 1.619,2.224, \quad 3.117,4.485,1.652$, $2.229, \quad 3.166,4.570, \quad 1.652, \quad 2.300,3.344,4.602, \quad 1.757,2.324,3.376,4.663$. Some descriptive statistics of these data are presented as follows. The minimum observed value is 0.040 , while the maximum value is 4.663 . The mean, median and variance are $2.563,2.385$, and 1.239 , respectively. Since the value of the mean is greater than that of variance, the aircraft windshield data is under-dispersed

Data set 2 is obtained from Smith and Naylor (1987), and consists of the strength of $1.5 \mathrm{~cm}$ glass fibres measured at the National Physical Laboratory, England.

Data Set 2: $0.55,0.74,0.77,0.81,0.84,1.24,0.93,1.04,1.11,1.13,1.30,1.25,1.27,1.28,1.29,1.48,1.36,1.39,1.42$, $1.48,1.51,1.49,1.49,1.50,1.50,1.55,1.52,1.53,1.54,1.55,1.61,1.58,1.59,1.60,1.61,1.63,1.61,1.61,1.62,1.62$, $1.67,1.64,1.66,1.66,1.66,1.70,1.68,1.68,1.69,1.70,1.78,1.73,1.76,1.76,1.77,1.89,1.81,1.82,1.84,1.84$, $2.00,2.01,2.24$. Some descriptive statistics of these data are presented as follows. The minimum observed value is 0.550 , while the maximum value is 2.240 . The mean, median and variance are $1.507,1.590$ and 0.1059 , respectively. Since the value of the mean is greater variance, the glass fibre data is said to be under-dispersed.

To demonstrate the tractability of $H P L$ distributions, Anderson-Darling $\left(A^{*}\right)$, Cramer-von Misses $(W)$, Probability value (P-value), Akaike information criterion (AIC), and Hannan-Quinn information criterion (HQIC) are calculated for each of the model considered. The selection procedure for the model that best fit the data is to choose the model having minimum value of these statistics except for the maximum value of the p-value. In this study, numerical results (of maximum likelihood estimates, and goodness of fit criteria) are calculated by using the goodness the Adequacypackage available in $\mathrm{R}$ language. The Adequacy package is also used to construct the Total Time on Test (TTT) plot for the two data sets to determine the shape of their hazard function and the Kernel density plot. Figure 6 shows that the two data set has increasing failure rate and figure 7 shows that the Air windshield data moderately skewed to the left and the glass fibre data also skewed to the same direction,

The $A^{*}, W, \mathrm{P}$-value, AIC, and HQIC are given for the sub-models $H L, P L, L$, and competitor models, the Kumaraswamy Generalized Power Lomax (KPL) by Nagarjuna, V. B. V. (2021), Kumaraswamy Generalized Lomax $(K L)$ distribution by Shams, T. M. (2013). Tables 3, 4 provide the estimated value of the parameters for data set 1 and 2 respectively. Table 4 and 6 shows the goodness of fit results for HPL distribution and competitor models for data set 1 and 2 respectively. These results show that the $H P L$ distribution is more appropriate than the other models considered since it possess the smallest AIC.BIC, HQIC, $A^{*}$ and the largest P-value

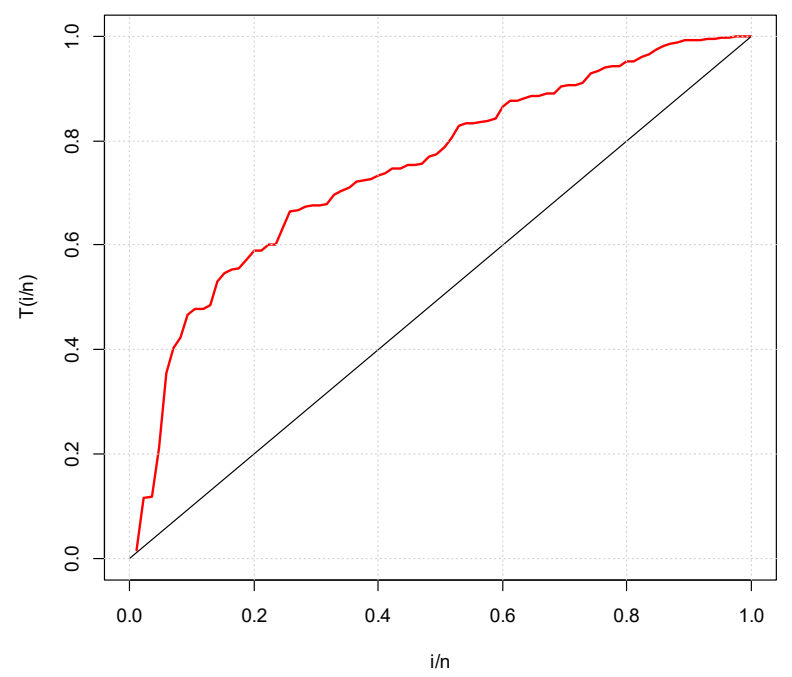

(i)

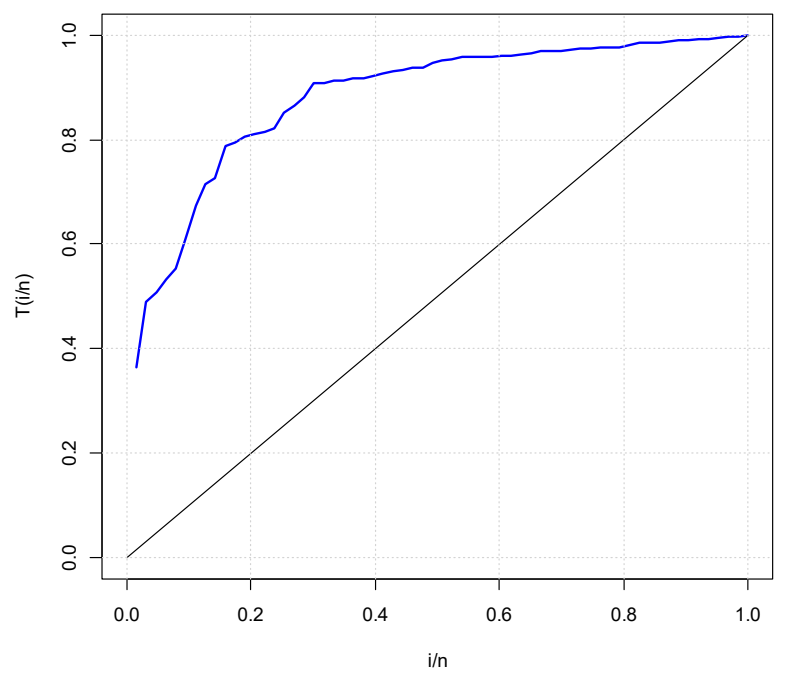

(ii) TTT plot for fibre glass data

Figure 6. TTT plot of the data 

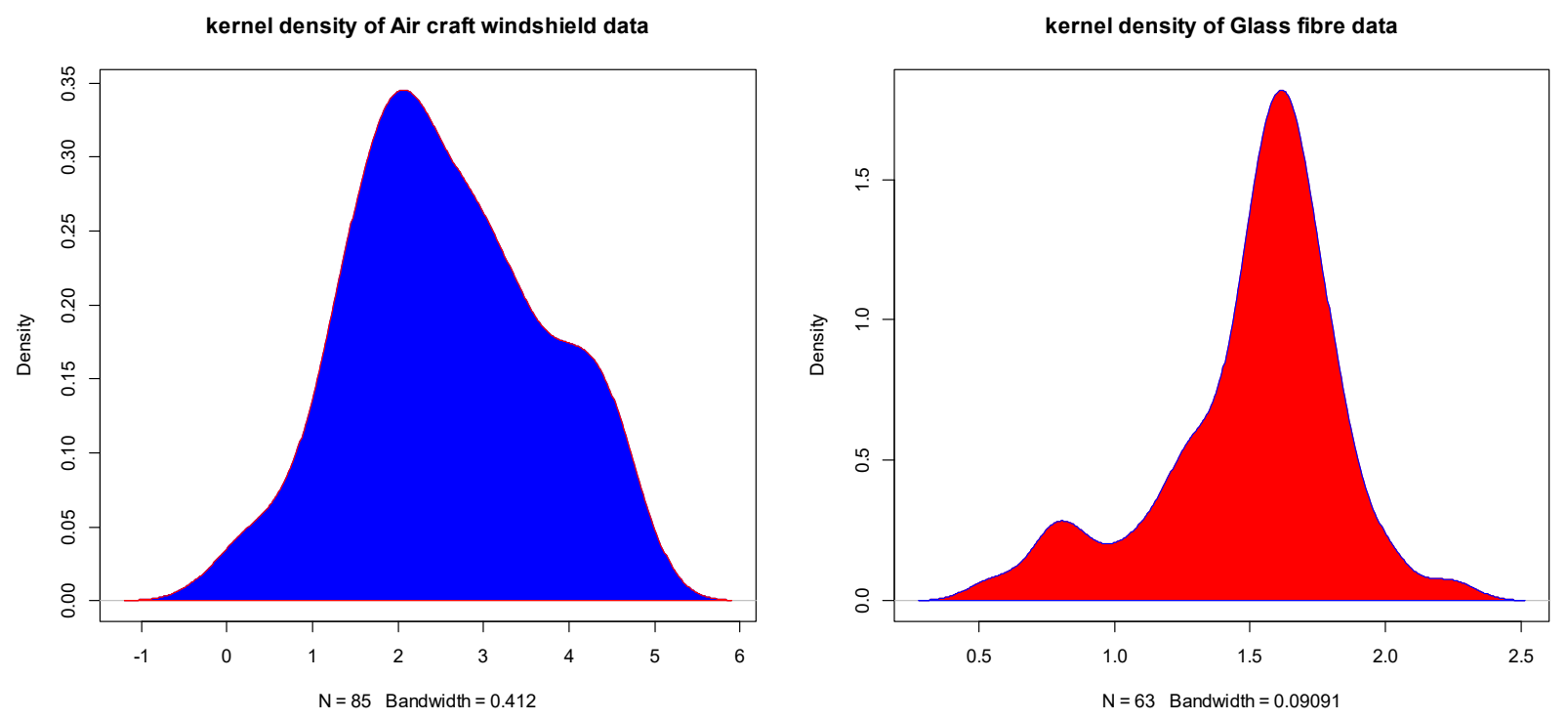

Figure 7. Kernel density for Windshield data Kernel density for Glass fibre data

Table 3. MLEs, Standard Error (SE) and Confidence interval in Curly Bracket for Aircraft Windshield data

\begin{tabular}{|c|c|c|c|c|c|}
\hline Model & $\widehat{\boldsymbol{a}}$ & $\widehat{\boldsymbol{b}}$ & $\widehat{\boldsymbol{c}}$ & $\widehat{\boldsymbol{k}}$ & $\widehat{\boldsymbol{v}}$ \\
\hline $\boldsymbol{H P L}$ & 0.6106 & 8.7811 & 8.3445 & 1.7339 & 13.3219 \\
& $(0.8960)$ & $(7.3612)$ & $(6.6743)$ & $(0.4348)$ & $(10.8966)$ \\
& $\{0.42,0.80\}$ & $\{7.14,10.29\}$ & $\{6.91,9.77\}$ & $\{1.64,1.83\}$ & $\{10.99,15.65\}$ \\
\hline $\boldsymbol{H L}$ & 0.8155 & - & 13.0356 & 27.0350 & 7.1015 \\
& $(0.3029)$ & $(-)$ & $(4.6990)$ & $(9.7039)$ & $(3.3180)$ \\
& $\{0.77,0.88\}$ & $\{-\}$ & $\{12.03,14.04\}$ & $\{24.96,29.11\}$ & $\{6.39,7.81\}$ \\
\hline KPL & 1.8974 & 3.5019 & 2.9135 & 1.5336 & 20.7448 \\
& $(0.9048)$ & $(1.9606)$ & $(1.7078)$ & $(0.5177)$ & $(15.5680)$ \\
& $\{1.70,2.09\}$ & $\{3.08,3.92\}$ & $\{2.55,3.28\}$ & $\{1.42,1.64\}$ & $\{17.42,24.07\}$ \\
\hline KL & 2.9760 & - & 2.8397 & 17.7832 & 15.6050 \\
& $(0.4887)$ & $(-)$ & $(1.7396)$ & $(10.0346)$ & $(12.8950)$ \\
& $\{2.87,3.08\}$ & $\{-\}$ & $\{2.47,3.21\}$ & $\{15.64,19.93\}$ & $\{12.85,18.36\}$ \\
\hline $\boldsymbol{P L}$ & 3.1360 & 2.5208 & - & 34.0794 & - \\
& $(1.3895)$ & $(0.2760)$ & $(-)$ & $(13.4667)$ & $(-)$ \\
& $\{2.84,3.43\}$ & $\{2.46,2.58\}$ & $\{-\}$ & $\{31.12,36.96\}$ & $\{-\}$ \\
\hline $\boldsymbol{L}$ & 9.0454 & 21.7786 & - & - & - \\
& $(0.4190)$ & $(38.4289)$ & $(-)$ & $(-)$ & $(-)$ \\
& $\{8.96,9.14\}$ & $\{13.56,29.99\}$ & $\{-\}$ & $\{-\}$ & $\{-\}$ \\
\hline
\end{tabular}

Table 4. Measures of goodness of fit for Aircraft Windshield data

\begin{tabular}{|c|c|c|c|c|c|c|}
\hline Model & $-\boldsymbol{l}$ & $\boldsymbol{A I C}$ & $\boldsymbol{H Q I C}$ & $\boldsymbol{A}^{*}$ & $\boldsymbol{W}$ & $\boldsymbol{p}-\boldsymbol{v a l u e}$ \\
\hline $\boldsymbol{H P L}$ & 130.209 & 270.419 & 275.332 & 0.5094 & 0.0563 & 0.8497 \\
\hline $\boldsymbol{H L}$ & 132.088 & 272.177 & 276.107 & 0.4673 & 0.0472 & 0.4438 \\
\hline $\boldsymbol{K P L}$ & 135.80 & 281.606 & 286.519 & 1.829069 & 0.2325 & $2.2 e-16$ \\
\hline $\boldsymbol{K} \boldsymbol{L}$ & 134.717 & 277.435 & 281.365 & 2.5636 & 0.3516 & $2.2 e-16$ \\
\hline $\boldsymbol{P L}$ & 134.762 & 275.524 & 278.472 & 0.8660 & 0.0901 & 0.4622 \\
\hline $\boldsymbol{L}$ & 168.791 & 341.582 & 343.547 & 0.1981 & 1.6068 & $1.10 e-07$ \\
\hline
\end{tabular}


Table 5. MLEs, Standard Error (SE) and Confidence interval in Curly Bracket for Glass Fibre data

\begin{tabular}{|c|c|c|c|c|c|}
\hline Model & $a$ & $\boldsymbol{b}$ & $c$ & $\boldsymbol{k}$ & $v$ \\
\hline HPL & $\begin{array}{c}0.1159 \\
(0.6904) \\
\{-0.06,0.29\}\end{array}$ & $\begin{array}{c}5.7044 \\
(3.2208) \\
\{4.91,6.50\}\end{array}$ & $\begin{array}{c}6.0754 \\
(3.5706) \\
\{5.19,6.96\}\end{array}$ & $\begin{array}{c}4.6366 \\
(0.8170) \\
\{4.44,4.84\}\end{array}$ & $\begin{array}{c}18.9588 \\
(11.7140) \\
\{16.07,21.85\}\end{array}$ \\
\hline$H L$ & $\begin{array}{c}1.3026 \\
(0.4379) \\
\{1.20,1.41\}\end{array}$ & $\begin{array}{l}- \\
(-) \\
\{-\}\end{array}$ & $\begin{array}{c}24.4218 \\
(9.8335) \\
\{21.99,26.85\}\end{array}$ & $\begin{array}{c}35.7293 \\
(11.1752) \\
\{32.97,38.49\}\end{array}$ & $\begin{array}{c}10.5724 \\
(5.4350) \\
\{9.23,11.92\}\end{array}$ \\
\hline$K P L$ & $\begin{array}{c}1.7729 \\
(1.0083) \\
\{1.52,2.02\}\end{array}$ & $\begin{array}{c}1.3910 \\
(1.5994) \\
\{1.00,1.79\}\end{array}$ & $\begin{array}{c}3.8967 \\
(1.9085) \\
\{3.43,4.37\}\end{array}$ & $\begin{array}{c}9.2834 \\
(10.2680) \\
\{6.75,11.82\}\end{array}$ & $\begin{array}{c}24.6763 \\
(20.9341) \\
\{19.51,29.85\}\end{array}$ \\
\hline$K L$ & $\begin{array}{c}12.5730 \\
(3.1215) \\
\{11.80,13.34\}\end{array}$ & $\begin{array}{l}- \\
(-) \\
\{-\}\end{array}$ & $\begin{array}{c}15.6930 \\
(20.2521) \\
\{10.69,20.69\}\end{array}$ & $\begin{array}{c}16.3790 \\
(12.751) \\
\{13.23,19.53\}\end{array}$ & $\begin{array}{c}14.9330 \\
(18.1862) \\
\{10.44,19.42\}\end{array}$ \\
\hline$P L$ & $\begin{array}{c}2.2852 \\
(0.8603) \\
\{2.07,2.50\}\end{array}$ & $\begin{array}{c}6.0218 \\
(0.7158) \\
\{5.85,6.20\}\end{array}$ & $\begin{array}{c}30.1575 \\
(11.0540) \\
\{27.43,32.89\}\end{array}$ & $\begin{array}{l}- \\
(-) \\
\{-\}\end{array}$ & $\begin{array}{l}- \\
(-) \\
\{-\}\end{array}$ \\
\hline$L$ & $\begin{array}{c}10.9251 \\
(\mathbf{4 . 8 5 7 3}) \\
\{9.73,12.13\}\end{array}$ & $\begin{array}{c}16.1369 \\
(\mathbf{7 . 4 0 1 1}) \\
\{14.31,17.97\}\end{array}$ & $\begin{array}{l}- \\
(-) \\
\{-\}\end{array}$ & $\begin{array}{l}- \\
(-) \\
\{-\}\end{array}$ & $\begin{array}{l}- \\
(-) \\
\{-\}\end{array}$ \\
\hline
\end{tabular}

Table 6. Measures of goodness of fit for failure Glass fibres data

\begin{tabular}{|c|c|c|c|c|c|c|}
\hline Model & $-\boldsymbol{l}$ & $\boldsymbol{A I C}$ & $\boldsymbol{H Q I C}$ & $\boldsymbol{A}^{*}$ & $\boldsymbol{W}$ & $\boldsymbol{p}-$ value \\
\hline $\boldsymbol{H P L}$ & 13.276 & 36.551 & 40.766 & 0.9788 & 0.1789 & 0.2369 \\
\hline $\boldsymbol{H L}$ & 41.832 & 91.664 & 95.036 & 2.4709 & 0.4481 & 0.0005 \\
\hline $\boldsymbol{K P} \boldsymbol{L}$ & 17.134 & 44.269 & 48.483 & 3.0047 & 0.5462 & $2.2 e-16$ \\
\hline $\boldsymbol{K} \boldsymbol{L}$ & 19.781 & 47.562 & 50.934 & 3.9256 & 0.7175 & $2.2 e-16$ \\
\hline $\boldsymbol{P L}$ & 18.974 & 43.947 & 46.476 & 123.5255 & 19.4425 & $2.2 e-16$ \\
\hline $\boldsymbol{L}$ & 91.515 & 187.030 & 188.715 & 121.7461 & 18.4913 & $2.2 e-16$ \\
\hline
\end{tabular}
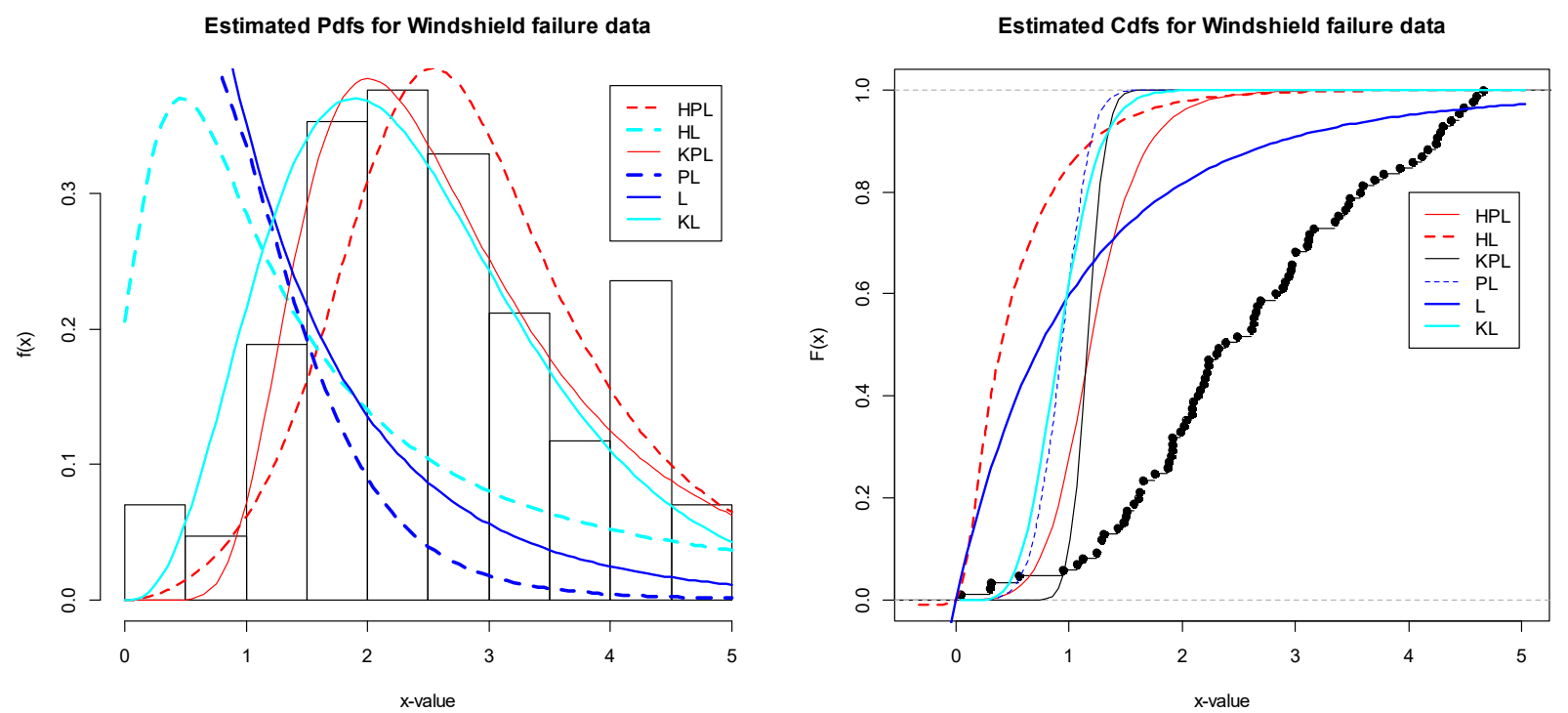

Figure 8. Estimated pdf and cdf function and other competing model for Air craft Windshield data 

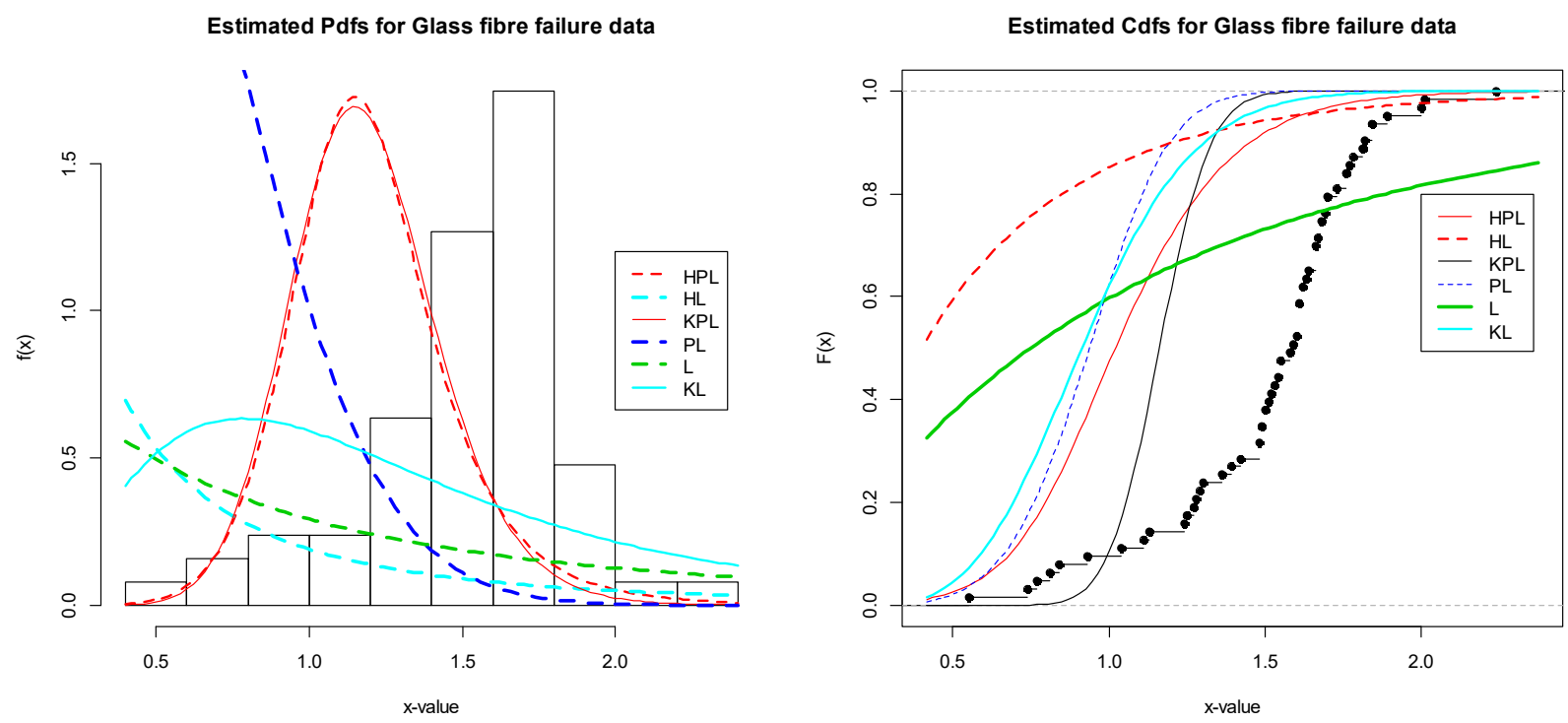

Figure 9. Estimated pdf and cdf function and other competing model for Glass fibre data

Based on Tables 4 and 6, we can conclude that HPL distribution provides the best fit and can therefore be considered the best model in the class of models considered. Figures 8 and 9 gives more information on the flexibility of $H P L$ distribution over its sub-models and other models considered.

\section{Concluding Remarks}

In this work, we have presented a new generalization of the $P L$ distribution, called the $H P L$ distribution. This generalization is obtained by transforming the three-parameter $P L$ model using Harris-G distribution as suggested by Aly and Benkherouf (2011). The properties of the proposed distribution are discussed. We obtain the analytical shapes of the density and hazard functions of the HPL distribution. We also consider mean deviations, Bonferroni and Lorenz curves, order statistics and Renyi entropy. Maximum likelihood estimation is discussed within the framework of asymptotic log-likelihood inferences including confidence intervals. The five parameter HPL distribution produced monotonically increasing, decreasing, and inverted bathtub hazard rates. In terms of the statistical significance of the model adequacy, the HPL distribution gives to a better fit than the it's sub-models and other competing models considered in this work

\section{Acknowledgements}

The authors would like to thank the anonymous referees for their valuable comments and suggestions which greatly improved the work.

\section{Funding}

This research is sponsored by the effort of the authors.

\section{Conflicts of Interest}

The authors declare no conflict of interest.

\section{References}

Abdul-Moniem, I. B., \& Abdel-Hameed, H. F. (2012). On the exponentiated Lomaxdistribution.International Journal of Mathematical Achieve, 3, 2144-2150.

Al. Zahrani, B., \& Sagor, H. (2014). The Poisson-lomax distribution. Rev. Colomb Estad., 37, $225-245$. https://doi.org/10.15446/rce.v37n1.44369

Aly, E., \& Benkherouf, L. (2011). A new family of distributions based on probability generating functions. Sankhya B-Applied interdisciplinary Statistics, 73(1), 70-80. https://doi.org/10.1007/s13571-011-0017-9

Alzaghal, A., \& Hamed, D. (2019). New families of Generalized Lomax distribution: Properties and Applications. International Journal of Statistics and Probability, 8, 51-68. https://doi.org/10.5539/ijsp.v8n6p51

Bryson, M. C. (1974). Heavy-tailed distribution: properties and tests. Technometrics, 16, 61-68. https://doi.org/10.1080/00401706.1974.10489150 
Cordeiro, G. M., Ortega, E. M., \& Popovic, B. V. (2015). The gamma Lomax distribution. Journal of Computer Simulation, 85, 305-319. https://doi.org/10.1080/00949655.2013.822869

El-Bassiouny, A. H., Abdo, N. F., \& Shahen, H. S. (2015). The Exponential Lomax distribution. International Journal of Computer Applications, 121, 24-29. https://doi.org/10.5120/21602-4713

Galton, F. (1883). Inquiries into Human Faculty and its Development.Macmillan and Company: London, UK. https://doi.org/10.1037/14178-000

Ghitany, M. E., Al-Awadhi, F. A., \& Alkhalfan, L. (2007). Marshall-Olkin extended Lomax distribution and its application to censored data. Communications in Statistics-Theory and Methods, 36(10), 1855-1866. https://doi.org/10.1080/03610920601126571

Harris, T. E. (1948). Branching processes. The Annals of Mathematical Statistics, 19(4), 474-494. https://doi.org/10.1214/aoms/1177730146

Kilany, N. M. (2016). Weighted Lomax distribution. SringerPlus, 5, 1862. https://doi.org/10.1186/s40064-016-3489-2

Lomax, K. S. (1954). Business failures: Another example of the analysis of failure data. Journal of American Statistical Association, 49, 847-852. https://doi.org/10.1080/01621459.1954.10501239

Nagarjuna, V. B. V., Vardhan, R. V., \& Chesneau, C. (2021). Kumaraswamy Generalized Power Lomax distribution and its Applications. Stats., 4, 28-45. https:/doi.org/10.3390/stats4010003

Pinho, L. G. B., Cordeiro, G. M., \& Nobre, J. S. (2015). On the Harris-G class of distributions: General results and applications. Brazillian Journal of Probability and Statistics, 29(4), 813-832. https://doi.org/10.1214/14-BJPS248

Rady, E. A., Hassaine, W. A., Elhaddad, T. A. (2016). The power Lomax distribution with an Application to bladder cancer data. SpringerPlus, 5, 1838. https://doi.org/10.1186/s40064-016-3464-y

Renyi, A. (1961). On measures of entropy and information. Proceedings of the 4th Berkeley Symposiumon Mathematical Statistics and Probability, vol.I, University of California Press, Berkeley. pp. 547-561.

Shams, T. M. (2013). The Kumaraswamy-Generalized Lomax distribution. Middle-East Journal of Science Research, 17, 641-646.

Simth, R. L., \& Naylor, J. C. (1987). A comparison of maximum likelihood and Bayesian estimators for three-parameter Weibull distribution. Applied Statistics, 36, 358-369.https://doi.org/10.2307/2347795

Tahir, M. H., Cordeiro, G. M., Mansoor, M., \& Zubair, M. (2015) The Weibull-Lomax distribution: Properties and Applications. Hacetep Journal of Mathematical Statistics, 44, 461-480. https://doi.org/10.15672/HJMS.2014147465

\section{Copyrights}

Copyright for this article is retained by the author(s), with first publication rights granted to the journal.

This is an open-access article distributed under the terms and conditions of the Creative Commons Attribution license (http://creativecommons.org/licenses/by/4.0/). 